Pacific Journal of Mathematic 


\title{
ON GROUP ACTIONS WITH NONZERO FIXED POINTS
}

\author{
J. W. JENKINS
}

Let $G$ be a locally compact group that acts continuously by linear transformations on a locally convex space $E$ and let $K$ be a compact convex subset of $E$ that is invariant under this action. In order to conclude that $K$ has a nonzero fixed point, it is necessary that both $G$ and $K$ satisfy certain conditions. With these assumptions on $K$, it is shown that the existence of nonzero fixed points is equivalent to polynomial growth on $G$, provided $G$ is connected or discrete, finitely generated and solvable.

A locally compact group $G$ is said to have the fixed point property if whenever $G$ acts continuously and linearly on a compact, convex subset $K$ of a locally convex space $E$ there is an element of $K$ invariant under the action of $G$. A well known result states that the fixed point property is equivalent to amenability (see [1]). If zero is an element of $K$, then, by the linearity of the action on $G$, it will be a fixed point, and amenability of $G$ does not gurantee the existence of any other fixed point. Of course, there may not be a nonzero fixed point. Consider, for example, $\boldsymbol{R}$ acting on $\boldsymbol{C}$ by multiplication by $e^{i t}$. The unit disk is a compact, convex, invariant subset and 0 is the only fixed point. Thus, in order to conclude the existence of a nonzero fixed point for a group action, it is clearly necessary to restrict the nature of the action. One such restriction, that exclude the example just cited, is to require that some half-space be invariant under the action. This is the essence of the first condition in Definition 1. This restriction alone, however, is still not sufficient to imply the existence of a nonzero fixed point. We consider another example. Let $C_{\infty}(\boldsymbol{R})$ denote the space of continuous functions on $\boldsymbol{R}$ that vanish at infinity equipped with the sup norm topology, and let $E$ be its dual space with the $w^{*}$-topology. Then $\boldsymbol{R}$ acts on $E$ by the contragradient to translation on $C_{\infty}(\boldsymbol{R})$. $K$, the set of positive linear functionals in $E$ of norm less than or equal one is compact, convex and lies in an invariant half-space. However, $K$ has no nonzero fixed point. In fact, one easily sees that for any $p \in K$

$$
w^{*} \lim _{t \rightarrow \pm \infty} t \cdot p=0,
$$

i.e., 0 is in the closure of every orbit in $K$. If we were to ignore this fact for a moment and try to construct a fixed point by the usual scheme, we would fix a nonzero element $p$ of $K$. Define a 
sequence of elements $p_{n}$ in $K$ by

$$
p_{n}=\frac{1}{2 n} \int_{-n}^{n} t \cdot p d t
$$

note that the $p_{n}$ 's are "nearly invariant", and use the compactness of $K$ to get an invariant cluster point of $\left\{p_{n}\right\}$. The difficulty, of course, is that $w^{*}-\lim p_{n}=0$. But even more critical is the fact that the entire orbits of the $p_{n}$ 's are " $w$-convergent to zero", i.e., for all $\varphi \in E^{*}$,

$$
\lim _{n \rightarrow \infty} \sup _{t \in \mathbf{R}}\left|\left\langle\varphi, t \cdot p_{n}\right\rangle\right|=0 .
$$

Hence, arbitrarily small neighborhoods of zero contain orbits of elements in $K$. The second condition in the following definition excludes such actions.

Definition 1. A locally compact group $G$ is said to have the nonzero fixed point property if whenever $G$ acts continuously and linearly on a locally convex space $E$ having an invariant, compact, convex subset $K$, and $K$ satisfies

(i) there is a $\varphi \in E^{*}$ so that $\langle\varphi, x\rangle>0$ for all $0 \neq x \in K$, and

(ii) for all $0 \neq x \in K, x / \sup _{G}\langle\varphi, s \cdot x\rangle \in K$, then $K$ has a nonzero fixed point.

The main result of this paper is the following characterization theorem.

THeORem 2. Suppose $G$ is a connected, locally compact group or a discrete, finitely generated, solvable group. Then $G$ has the nonzero fixed point property if, and only if, $G$ has polynomial growth.

The proof will be given in a series of propositions, but first we recall some facts about groups with polynomial growth.

Given a locally compact group $G$, and measurable subset $U$, denote by $|U|$ the left Haar measure of $U$, and for a positive integer $n$ set $U^{n}=\left\{u_{1} u_{2} \cdots u_{n} \mid u_{i} \in U, 1 \leqq i \leqq n\right\} . \quad G$ is said to have polynomial growth if for any compact neighborhood $U$ of the identity in $G$, there is a polynomial $p$ such that $\left|U^{n}\right| \leqq p(n)$ for all $n=1,2, \cdots$.

Milnor [7] and Wolf [9] showed that a discrete, finitely generated, solvable group $G$ has polynomial growth if, and only if, it contains a nilpotent subgroup with finite index. Rosenblatt [8] added to the characterization by showing that for such $G$, polynomial growth is equivalent to $G$ not having a free noncommutative subsemigroup on two generators. 
For a Lie group $G$, let $t \rightarrow \operatorname{Ad} t$ denote the adjoint representation of $G$ on its Lie algebra. $G$ is said to be type $R$ if the eigenvalues of Ad $t$ are of modulus one for all $t \in G$. A connected, locally compact group $G$ is said to be type $R$ if for some compact, normal subgroup $K, G / K$ is a type $R$ Lie group.

A subset $S$ of a locally compact group $G$ is said to be uniformly discrete if there is a neighborhood $U$ of the identity in $G$ such that $s U \cap t U=\varnothing$ for $s, t \in S, s \neq t$.

It is shown in Jenkins [5] that for a connected group, $G$, polynomial growth is equivalent to $G$ being type $R$, and also equivalent to $G$ not having a uniformly discrete free subsemigroup on two generators. (The first equivalence was also proven by Guivarc'h [2].)

The following lemma was first proved in Guivarc'h [2]. We include a proof here for the convenience of the reader.

LemMA 3. Suppose $G$ is a conncted, locally compact group or a discrete, finitely generated, solvable group. If $G$ has polynomial growth, then there is a normal series $\{e\}=G_{0} \subset G_{1} \subset \cdots \subset G_{n}=G$ such that every element of $G_{j+1} / G_{j}$ is contained in a compact neighborhood of the identity that is invariant under the inner-automorphisms from $G / G_{j}$.

Proof. If $G$ contains a normal, nilpotent subgroup of finite index, $N$, then the lower central series of $N$ will give the desired normal series of $G$.

Suppose $G$ is a connected Lie group with polynomial growth. Let $S$ be the solv-radical of $G, L S$ its Lie algebra, and $L S_{c}$, the complexification of $L S$. By Lie's theorem, there is an ordered basis for $L S_{c},\left\{X_{1}, \cdots, X_{m}\right\}$ so that the matrix representation for Ad $s$ with respect to this basis is upper triangular for all $s \in S$. Let $V_{j}$ be the subspace spanned by $\left\{X_{1}, \cdots, X_{j}\right\}$. Then, because of polynomial growth, $S$ is type $R$, and the action of $\mathrm{Ad} s$ on $V_{j+1} / V_{j}$ is multiplication by a complex number of modulus one, i.e., Ad $s$ acts by rotation on $V_{j+1} / V_{j}$. Thus we can find subspaces $\{0\}=W_{0} \subset W_{1} \subset \cdots \subset$ $W_{n}=L S$, the real Lie algebra of $S$, each invariant under Ad $s$, for all $s \in S$, with $\operatorname{dim}\left(W_{j+1} / W_{j}\right) \leqq 2$, and with Ad $s$ actaing by rotation of $W_{j+1} / W_{j}$. Thus $W_{j}$ is an ideal in $L S$ and if $S_{j}$ is the corresponding closed, normal subgroup of $S$, each element of $S_{j+1} / S_{j}$ is in a compact neighborhood that is invariant under the inner-automorphisms from $S / S_{j}$. Since $G$ has polynomial growth, the semisimple part of $G, G / S$, is compact. Hence, the $S_{j}$ 's give the desired normal series for $G$.

If $G$ is a connected group with polynomial growth, there is a 
compact, normal subgroup $K$ so that $G / K$ is a Lie group with polynomial growth. The above argument applied to $G / K$ completes the proof.

The following proposition, which is the keystone in the proof of Theorem 2, was inspired by Ludwig [6].

Proposition 4. Let $G$ be a locally compact group with polynomial growth, and suppose $G$ acts continuously and linearly on a locally convex space $E$. Let $K$ be a compact, convex subset of $E$ that satisfies the conditions in Definition 1 . Let $L, H$ be normal subgroups of $G$ with $L \subset H$, and suppose every element of $H / L$ is contained in a compact, $G / L$ invariant neighborhood. If $K$ has a nonzero point fixed by $L$, it has a nonzero point fixed by $H$.

Proof. Let $\varepsilon>0$ and $U$, a compact, symmetric neighborhood of the identity in $H / L$ that is invariant under the inner-automorphisms from $G / L$, be given. We define a function $f_{\varepsilon, \dot{U}}$ on $H / L$ by $f_{\varepsilon, \dot{U}}(\dot{s})=$ $(1+\varepsilon)^{-1}$ if $\dot{s} \in \dot{U}, f_{\varepsilon, \dot{U}}(\dot{s})=(1+\varepsilon)^{-n}$ if $\dot{s} \in \dot{U}^{n} \sim \dot{U}^{n-1}$ for $n \geqq 2$, and $f_{\varepsilon, \dot{U}}(\dot{s})=0$ if $\dot{s} \notin\langle\dot{U}\rangle$, the subgroup generated by $\dot{U}$. Since $H / L$ has polynomial growth

$$
\left\|f_{\varepsilon, \dot{U}}\right\|_{1}=(1+\varepsilon)^{-1}|\dot{U}|+\sum_{n=2}^{\infty}(1+\varepsilon)^{-n}\left|\dot{U}^{n} \sim \dot{U}^{n-1}\right|<\infty .
$$

Also note that for $\dot{s} \in \dot{U}$ and $\dot{t} \in\langle\dot{U}\rangle$

$$
\left|f_{\varepsilon, \dot{U}}(\dot{s} \dot{t})-f_{\dot{\varepsilon}, \dot{U}}(\dot{t})\right| \leqq \varepsilon f_{\varepsilon, \dot{U}}(\dot{t}) .
$$

Let $0 \neq x_{0} \in K$ such that $s \cdot x_{0}=x_{0}$ for all $s \in L$. Then $H / L$ acts on $x_{0}$ and we can define $x_{\varepsilon, \dot{U}}^{\prime} \in E^{* *}$ by setting

$$
\left\langle x_{\varepsilon, \dot{U}}^{\prime}, \psi\right\rangle=\int_{H / L} f_{\varepsilon, \dot{U}}(\dot{s})\left\langle\dot{s} \cdot x_{0}, \psi\right\rangle d s,
$$

for each $\psi \in E^{*}$. Since $K$ is compact and convex, $x_{\varepsilon, \dot{v}}^{\prime}$ is in the canonical image of $K$ in $E^{* *}$. We denote its preimage in $K$ also by $x_{\varepsilon, \dot{U}}^{\prime}$.

Let $\varphi \in E^{*}$ as in Definition 1, i.e., $\langle\varphi, x\rangle>0$ and $x / \sup _{s \in G}\langle\varphi, s \cdot x\rangle \in K$ for all $0 \neq x \in K$. Define

$$
\alpha_{\varepsilon, \dot{U}}=\sup _{s \in \dot{G}}\left\langle\varphi, s \cdot x_{\varepsilon, \dot{U}}^{\prime}\right\rangle,
$$

and pick $a \in G$ so that

$$
\left\langle\varphi, a \cdot x_{\varepsilon, \dot{U}}^{\prime}\right\rangle \geqq(1-\varepsilon) \alpha_{\varepsilon, \dot{U}} \cdot
$$

Finally, define

$$
x_{\varepsilon, \dot{U}}=\alpha_{\varepsilon, \dot{U}}^{-1} a \cdot x_{\varepsilon, \dot{U}}^{\prime} .
$$


Note that by condition (ii) of Definition $1, x_{\varepsilon, \dot{U}} \in K$, since a real multiple of $x_{\varepsilon, \dot{u}}^{\prime} \in K$.

Let $\psi \in E^{*}$ and $\dot{b} \in \dot{U}$.

$$
\begin{aligned}
\left|\left\langle\psi, b \cdot x_{\varepsilon, \dot{U}}-x_{\varepsilon, \dot{U}}\right\rangle\right| & =\alpha_{\varepsilon, \dot{U}}^{-1}\left|\int f_{\varepsilon, \dot{U}}(\dot{s})\left(\left\langle\psi, b a s \cdot x_{0}\right\rangle-\left\langle\psi, a s \cdot x_{0}\right\rangle\right) d \dot{s}\right| \\
& \leqq \alpha_{\varepsilon, \dot{U}}^{-1} \int\left|f_{\varepsilon, \dot{U}}\left(\dot{a}^{-1} \dot{b}^{-1} \dot{a} \dot{s}\right)-f_{\varepsilon, \dot{U}}(\dot{s})\right|\left|\left\langle\psi, \dot{a} \dot{s} \cdot x_{0}\right\rangle\right| d \dot{s} \\
& \leqq \varepsilon \alpha_{\varepsilon, \dot{U}}^{-1} \int f_{\varepsilon, \dot{U}}(\dot{s})\left|\left\langle\dot{\psi}, \dot{a} \dot{s} \cdot x_{0}\right\rangle\right| d \dot{s} .
\end{aligned}
$$

Thus, in particular,

$$
\left|\left\langle\varphi, \dot{b} \cdot x_{\varepsilon, \dot{U}}-x_{\varepsilon, \dot{U}}\right\rangle\right| \leqq \varepsilon .
$$

We consider the net $\left\{x_{\varepsilon, \dot{U}}\right\}$ ordered by $(\varepsilon, \dot{U}) \leqq\left(\varepsilon^{\prime}, \dot{U}^{\prime}\right)$ if $\varepsilon<\varepsilon^{\prime}$ and $\dot{U}^{\prime} \subseteq \dot{U}$, and pick a cluster point $x_{\infty}$. Note that $x_{\infty} \in K$, that $s \cdot x_{\infty}=x_{\infty}$ for all $s \in L$, and that $\left\langle\varphi, x \cdot x_{\infty}\right\rangle=1$ for all $s \in L$. Thus we may assume that the original $x_{0}$ had its orbit in the hyperplane $\{y \mid\langle\varphi, y\rangle=1\}$. But then $\alpha_{\varepsilon, \dot{U}} \geqq\left\|f_{\varepsilon, \dot{U}}\right\|_{1}$, and we have that for all $\psi \in E^{*}$ and $\dot{b} \in \dot{U}$

$$
\left|\left\langle\dot{\psi}, \dot{b} \cdot x_{\varepsilon, \dot{C}}-x_{\varepsilon, \dot{U}}\right\rangle\right| \leqq \varepsilon \sup _{s \in \dot{G}}\left|\left\langle\dot{\psi}, s \cdot x_{0}\right\rangle\right| .
$$

Thus, for all $s \in H$ and $\psi \in E^{*}$

$$
\left\langle\psi, s \cdot x_{\infty}\right\rangle=\left\langle\psi, x_{\infty}\right\rangle
$$

Hence $s \cdot x_{\infty}=x_{\infty}$ for all $s \in H$.

Lemma 3 and Proposition 4 combine to prove that polynomial growth is sufficient to give the nonzero fixed point property for the class of groups given in Theorem 2. In order to prove the converse we need the following proposition which has some interesting consequences of its own.

Proposition 5. Let $G$ be a locally compact, $\sigma$-compact group that has the nonzero fixed point property. Let $\theta \in B C(G)$, the bounded, continuous functions on $G$, with $0 \neq \theta \geqq 0$ and $\theta \leqq f_{0} * \theta$ for some $f_{0} \in L^{1}(G)$.

Let

$$
L_{0}=\left\{\psi^{\prime} \in L^{\infty}(G)|| \psi \mid \leqq f * \theta \text { some } f \in L^{1}(G)\right\}
$$

and set

$$
\|\psi\|_{\theta}=\inf \left\{\|f\|_{1} \| \psi \mid \leqq f * \theta\right\}
$$

Then there is a continuous, positive linear functional on $L_{\theta}, p$, such that $\langle p, s \cdot \psi\rangle=\langle p, \psi\rangle$ all $s \in G$ and $\psi \in L_{\theta}$ and such that $\langle p, \theta\rangle=1$. 
Proof. First note that we may assume that $\theta \leqq f_{0} * \theta$ where $f_{0}(s)>0$ for almost all $s \in G$.

Let

$$
L_{\theta}^{0}=\left\{f * \theta \mid f \in L^{1}(G)\right\}
$$

and let $E$ denote the dual of $L_{\theta}^{0}$ with the $w^{*}$-topology. $G$ acts on $E$ by

$$
\langle s \cdot p, f * \theta\rangle=\langle p, s f * \theta\rangle
$$

where $s f(t)=f(s t)$ for all $s, t \in G$ and $f \in L^{1}(G)$. This action is continuous since

$$
\begin{aligned}
|\langle s \cdot p, f * \theta\rangle-\langle p, f * \theta\rangle| & \leqq|\langle p,(s f-f) * \theta\rangle| \\
& \leqq\|p\|\|s f-f\|_{1},
\end{aligned}
$$

where $\|p\|$ is the norm of $p$ induced by $\|\cdot\|_{\theta}$ restricted to $L_{\theta}^{0}$.

Let

$$
K=\{p \in E \mid p \geqq 0,\|p\| \leqq 1\} .
$$

Then $K$ is compact, convex and satisfies the two conditions of Definition 1.

The first condition is satisfied by the linear functional $f_{0} * \theta$, for if $p \in K$ and $\left\langle p, f_{0} * \theta\right\rangle=0$ then for all $g \in L^{1}(G)$ with $0 \leqq g \leqq f_{0}$, $\langle p, g * \theta\rangle=0$. Thus, for all bounded, compactly supported $g$ in $L^{1}(G)$, $\langle p, g * \theta\rangle=0$. By denseness of the set of all such $g * \theta$ in $L_{\theta}^{0}, p=0$.

For the second condition, let $\psi \in L_{\theta}^{0}$ and $p \in K$. Then, if $|\psi| \leqq$ $f * \theta$

$$
\begin{aligned}
|\langle p, \psi\rangle| & \leqq\langle p,|\psi|\rangle \leqq\left\langle p, f * f_{0} * \theta\right\rangle \\
& =\int f(s)\left\langle p, s f_{0} * \theta\right\rangle d s .
\end{aligned}
$$

Thus, setting

$$
\alpha=\sup _{s \in G}\left\langle p, s f_{0} * \theta\right\rangle,
$$

we have that for $|\psi| \leqq f * \theta$,

$$
|\langle p, \psi\rangle| \leqq \alpha\|f\|_{1}
$$

Hence

$$
\|p\|=\sup _{\|\psi\|_{\theta}=1}|\langle p, \psi\rangle| \leqq \alpha
$$

and so $p / \alpha \in K$.

An application of the nonzero fixed point property gives a $0 \neq p^{\prime} \in K$ that is fixed by $G$.

Let $\mathscr{C}$ be a neighborhood basis of $e$ in $G$ consisting of open sets with compact closures. For each $U \in \mathscr{\mathscr { C }}$, let $\varphi_{U}$ denote the normalized 
characteristic function of $U$. Assume $\lim \inf _{\mathscr{U}}\left\langle p^{\prime}, \varphi_{U} * \theta\right\rangle=0$. Then, by relabeling, we may assume $\lim _{\mathscr{t}}\left\langle p^{\prime}, \varphi_{U} * \theta\right\rangle=0$. Fix a $U_{0} \in \mathscr{C}$. Given $\varepsilon>0$ there exists $s_{1}, \cdots, s_{n} \in G, U_{1}, \cdots, U_{n} \in \mathscr{C}$ such that

(i) $s_{i} U_{i} \cap s_{j} U_{j}=\varnothing$ for $i \neq j$.

(ii) $\chi_{U_{0}} \leqq \sum_{k=1}^{n} s_{k} \cdot \varphi_{U_{k}}\left|U_{k}\right|$.

(iii) ||$\chi_{U_{0}}-\sum s_{k} \cdot \varphi_{U_{k}}\left|U_{k}\right| \|_{1} \leqq \varepsilon$.

(iv) $\left\langle p^{\prime}, \varphi_{U_{i}} * \theta\right\rangle \leqq \varepsilon$.

Thus,

$$
0 \leqq\left\langle p^{\prime}, \chi_{U_{0}} * \theta\right\rangle \leqq\left\langle p^{\prime}, \sum s_{k} \cdot \varphi_{U_{k}}\left|U_{k}\right|\right\rangle \leqq \varepsilon \sum_{k=1}^{n}\left|U_{k}\right| \leqq \varepsilon\left(\varepsilon+\left|U_{0}\right|\right) .
$$

Since $\varepsilon>0$ was arbitrary, $\left\langle p^{\prime}, \chi_{L_{0}^{*}} * \theta\right\rangle=0$. It follows immediately that $p^{\prime}=0$. This contradiction implies that $\lim \inf _{\mathscr{r}}\left\langle p^{\prime}, \varphi_{u} * \theta\right\rangle=$ $\delta>0$, and from this, one easily sees that

$$
\left\langle p^{\prime}, f * \theta\right\rangle=\delta \int f(s) d s
$$

for all $f \in L^{1}(G)$.

To get the desired element in $L_{\theta}^{*}$, we note first that by the nonzero fixed point property $G$ is amenable. Pick a left invariant mean $m$ on $L^{\infty}(G)$, extend $p^{\prime}$ to a continuous, positive linear functional $\bar{p}$ on $L_{0}$ by the Krein Extension Theorem, and define $p$ on $L_{\theta}$ by $\langle p, \psi\rangle=\left\langle m, F_{\psi}\right\rangle$ where $F_{\psi}(s)=\langle\bar{p}, s \psi\rangle$. One easily checks that, up to normalization, $p$ is the desired functional.

REMaRK. In [8], Rosenblatt defined a group $G$ to be superamenable if for any measurable subset $A \subset G$ there is a translation invariant, positive linear functional $p$ defined on the space spanned by the left-translates of $\chi_{A}$ with $\left\langle p, \chi_{A}\right\rangle=1$. He showed that a discrete group with polynomial growth is superamenable. In [4], this was generalized by showing that for any locally compact group $G$ with polynomial growth and $0 \neq \theta \in L^{\infty}(G), \theta \geqq 0$, there is a translation invariant, positive linear functional $p$ defined on the space spanned by the left-translates of $\theta$ with $\langle p, \theta\rangle=1$. However, this functional $p$, in general, can not be extended to the larger space $L_{\theta}$ (see the remark after Corollary 6.).

A Banach *-algebra $A$ is said to be symmetric if for all $a \in A$, $\operatorname{sp}\left(a a^{*}\right) \subseteq[0, \infty)$. Ludwig [6] proved that if $G$ is a connected locally compact group or a discrete, finitely generated, solvable group and $G$ has polynomial growth then $L^{1}(G)$ is symmetric. Essentially, the same proof gives

CoRollary 6. If $G$ has the nonzero fixed point property then $L^{1}(G)$ is symmetric. 
Proof. Suppose $f_{0} \in L(G)$ such that $-1 \in \mathbf{s p}\left(f_{0} * f_{0}^{*}\right)$. Then there is an $0 \neq \varphi \in B C(G)$ such that $f_{0} * f_{0}^{*} * \phi=-\varphi$. Let

$$
\theta=|\varphi|^{2} \text {. }
$$

Note that

$$
\theta=|\varphi|^{2}=\left|f_{0} * f_{0}^{*} * \varphi\right|^{2} \leqq \| f_{0} * f_{0}^{*}||_{1}\left|f_{0} * f_{0}^{*}\right| * \theta
$$

Hence, by Proposition 5, there is a positive continuous linear function $p$ on $L_{0}$ that is invariant and has $\langle p, \theta\rangle=1$.

Define a form $B$ on $L^{1}(G)$ by

$$
B(f, g)=\langle p,(f * \varphi)(\overline{g * \varphi})\rangle .
$$

This makes sense, for if $f, g \in L_{1}(G)$ then

$$
|(f * \phi)(\overline{g * \phi})| \leqq \frac{1}{2}\left\{|g * \phi|^{2}+|f * \varphi|^{2}\right\} \in L_{\theta} .
$$

Clearly $B(f, f) \geqq 0$ for all $f \in L(G)$ and $B\left(f_{0} * f_{0}^{*}, f_{0} * f_{0}^{*}\right)=\left\langle p,|\varphi|^{2}\right\rangle=1$. Also $B$ is bounded, since for $g \in L^{1}(G)$

$$
B(g, g)=\left\langle p,|g * \varphi|^{2}\right\rangle \leqq\|g\|_{1}\langle p,|g| * \theta\rangle=\|g\|_{1}^{2} .
$$

Finally, by the invariance of $p, B(s f, s g)=B(f, g)$ for all $f, g \in L^{\prime}(G)$ and $s \in G$. Thus $B(f * g, h)=B\left(g, f^{*} * h\right)$. Hence

$$
0 \leqq B\left(f_{0}^{*}, f_{0}^{*}\right)=-B\left(f_{0}^{*} * f_{0}^{*} * f_{0}^{*}, f_{0}^{*}\right)=-1 \text {. }
$$

This contradiction implies that $-1 \notin \mathrm{sp}\left(f_{0} * f_{0}^{*}\right)$.

REMARK. Hulanicki [3] has shown that there is a discrete, solvable group (not finitely generated) with polynomial growth that has a nonsymmetric group algebra. Hence not every group with polynomial growth enjoys the nonzero fixed point property.

Proposition 7. Suppose $G$ is a connected locally compact group or a discrete, finitely generated, solvable group. If $G$ does not have polynomial growth it does not have the nonzero fixed point property.

Proof. With the assumptions on $G$, failure of polynomial growth implies the existence of elements $a, b \in G$ and a compact neighborhood of the identity $U$ such that the semigroup generated by $a$ and $b$, $S$, is free and for $s, t \in S, s \neq t, s U \cap t U=\varnothing$. Let $\psi$ be the characteristic function of $S \cdot U$. Let $V$ be a compact neighborhood of the identity and $\alpha>0$ such that $\chi_{V} \leqq \alpha \chi_{V} * \chi_{V}$. Then, with $f=\alpha \chi_{V}$ and $\theta=\chi_{V} * \psi$, we have $\theta \leqq f * \theta$. Hence, if $G$ has the nonzero fixed point property, Proposition 5 gives a positive linear function $p \in L_{\theta}^{*}$ that is invariant and such that $\langle p, \theta\rangle=1$. Now $\psi \in L_{\theta}$ and 


$$
\langle p, \theta\rangle=\int_{V}\langle p s, s \cdot \psi\rangle d=|V|\langle p, \psi\rangle>0
$$

Since $S$ is free and uniformly discrete, $a S U \cap b S U=\varnothing$. Hence $\psi-a \cdot \psi-b \cdot \psi \geqq 0$. Thus, since $p \geqq 0$ and invariant, $\langle p, \psi\rangle \geqq 2\langle p, \psi\rangle$. This contradiction shows that $G$ does not have the nonzero fixed point property.

Acknowledgment. This author is indebted to Professor P. Eymard for several conversations which inspired this paper.

\section{REFERENCES}

1. F.P. Greenleaf, Invariant Means on Topological Groups, Van Nostrand, New York, 1969.

2. Y. Guivarc'h, Croissance polynomiale et periodes des fonctions harmoniques, Bull. Soc. Math. France, 101 (1973), 333-379.

3. A. Hulanicki, A solvable group with polynomial growth and non-symmetric group algebra, preprint.

4. J. Jenkins, Folner's condition for groups with polynomial growth, Math. Scand., 35 (1974).

5. - Growth of locally compact groups, J. Functional Analysis, 12 (1973), 113-127.

6. J. Ludwig, A class of symmetric and a class of Wiener group algebras, preprint. 7. J. Milnor, Growth of finitely generated solvable groups, J. of Diff. Geometry, 2 (1968), 447-449.

8. J. Rosenblatt, Invariant measures and growth conditions, Trans. Amer. Math. Soc., 193 (1974), 33-53.

9. J. Wolf, Growth of finitely generated solvable groups, J. of Diff. Geometry, 2 (1968), 421-446.

Received October 5, 1979. This research was partially supported by National Science Foundation grant MCS-7702045.

State University of New York at Albany

ALBANY, NY 12222 



\section{PACIFIC JOURNAL OF MATHEMATICS}

\section{EDITORS}

DONALD BABBITT (Managing Editor)

University of California

Los Angeles, CA 90024

Hugo RossI

University of Utah

Salt Lake City, UT 84112

C. C. MOORE and ANDREW OGG

University of California

Berkeley, CA 94720

\section{J. DugundjI}

Department of Mathematics

University of Southern California

Los Angeles, CA 90007

R. FinN and J. Milgram

Stanford University

Stanford, CA 94305

\section{ASSOCIATE EDITORS}
E. F. BeCKENBACH
B. H. Neumann
F. WoLF
K. YoSHIDA

\section{SUPPORTING INSTITUTIONS}

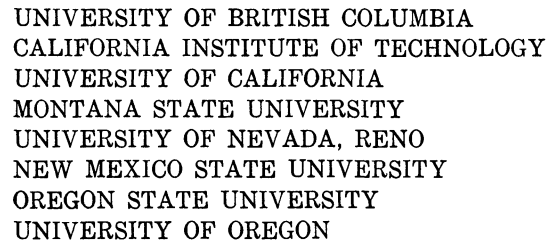

UNIVERSITY OF BRITISH COLUMBIA CALIFORNIA INSTITUTE OF TECHNOLOGY UNIVERSITY OF CALIFORNIA MONTANA STATE UNIVERSITY UNIVERSITY OF NEVADA, RENO NEW MEXICO STATE UNIVERSITY OREGON STATE UNIVERSITY UNIVERSITY OF OREGON

\author{
UNIVERSITY OF SOUTHERN CALIFORNIA \\ STANFORD UNIVERSITY \\ UNIVERSITY OF HAWAII \\ UNIVERSITY OF TOKYO \\ UNIVERSITY OF UTAH \\ WASHINGTON STATE UNIVERSITY \\ UNIVERSITY OF WASHINGTON
}

The Supporting Institutions listed above contribute to the cost of publication of this Journal, but they are not owners or publishers and have no responsibility for its content or policies.

Mathematical papers intended for publication in the Pacific Journal of Mathematics should be in typed form or offset-reproduced, (not dittoed), double spaced with large margins. Please do not use built up fractions in the text of the manuscript. However, you may use them in the displayed equations. Underline Greek letters in red, German in green, and script in blue. The first paragraph or two must be capable of being used separately as a synopsis of the entire paper. Please propose a heading for the odd numbered pages of less than 35 characters. Manuscripts, in triplicate, may be sent to any one of the editors. Please classify according to the scheme of Math. Reviews, Index to Vol. 39. Supply name and address of author to whom proofs should be sent. All other communications should be addressed to the managing editor, or Elaine Barth, University of California, Los Angeles, California, 90024.

50 reprints to each author are provided free for each article, only if page charges have been substantially paid. Additional copies may be obtained at cost in multiples of 50 .

The Pacific Journal of Mathematics is issued monthly as of January 1966. Regular subscription rate: $\$ 84.00$ a year (6 Vols., 12 issues). Special rate: $\$ 42.00$ a year to individual members of supporting institutions.

Subscriptions, orders for numbers issued in the last three calendar years, and changes of address shoud be sent to Pacific Journal of Mathematics, P.O. Box 969, Carmel Valley, CA 93924, U.S.A Old back numbers obtainable from Kraus Periodicals Co., Route 100, Millwood, NY 10546.

PUBLISHED BY PACIFIC JOURNAL OF MATHEMATICS, A NON-PROFIT CORPORATION

Printed at Kokusai Bunken Insatsusha (International Academic Printing Co., Ltd.). 8-8, 3-chome, Takadanobaba, Shinjuku-ku, Tokyo 160, Japan. 


\section{Pacific Journal of Mathematics}

\section{Vol. 91, No. 2 December, 1980}

Victor P. Camillo and Julius Martin Zelmanowitz, Dimension modules ... . . 249

Yonina S. Cooper, Stable sequences in pre-abelian categories ........... 263

Chandrakant Mahadeorao Deo and H. Ship-Fah Wong, On Berry-Esseen approximation and a functional LIL for a class of dependent random fields.........................................

H. P. Dikshit and S. N. Dubey, $|C, 1|$ summability of series associated with

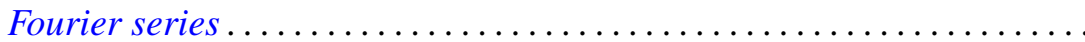

M. Edelstein, On the homomorphic and isomorphic embeddings of a semiflow into a radial flow.

Gilles Godefroy, Compacts de Rosenthal ..................... 293

James Guyker, Commuting hyponormal operators ................ 307

Thomas Eric Hall and Peter R. Jones, On the lattice of varieties of bands of

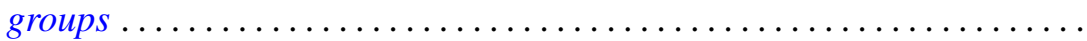

Taqdir Husain and Saleem H. Watson, Topological algebras with orthogonal Schauder bases ....................................

V. K. Jain, Some expansions involving basic hypergeometric functions of two variables. . .

Joe W. Jenkins, On group actions with nonzero fixed points ........... 363

Michael Ellsworth Mays, Groups of square-free order are scarce ........ 373

Michael John McAsey, Canonical models for invariant subspaces... 377

Peter A. McCoy, Singularities of solutions to linear second order elliptic partial differential equations with analytic coefficients by approximation methods...

Terrence Millar, Homogeneous models and decidability.

Stephen Carl Milne, A multiple series transformation of the very well poised

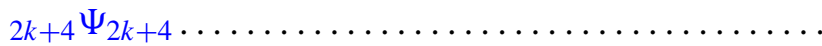

Robert Olin and James E. Thomson, Irreducible operators whose spectra are spectral sets...

Robert John Piacenza, Cohomology of diagrams and equivariant singular

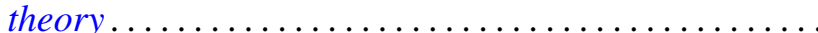

Louis Jackson Ratliff, Jr., Integrally closed ideals and asymptotic prime divisors

Robert Breckenridge Warfield, Jr., Cancellation of modules and groups and stable range of endomorphism rings.................

B. J. Day, Correction to: "Locale geometry" ...............

Stanley Stephen Page, Correction to: "Regular FPF rings" ... 